\title{
Continuity of the density of states in a magnetic field $\dagger$
}

\author{
Barry Simon \\ Departments of Mathematics and Physics, California Institute of Technology, Pasadena, \\ California 91125 , USA
}

Received 22 March 1982

\begin{abstract}
Fix a periodic potential, $V$, and let $k(E, B)$ be the integrated density of states up to energy $E$ in a constant magnetic field $B$. We prove that $k(E, B)$ is continuous in $B$ at all points where it is continuous in $E$. We prove a similar result when $V$ is zero and $B$ is a multiple of a periodic magnetic field.
\end{abstract}

It is well known that there is an analogy between the Hamiltonian operator of a solid in constant magnetic field (Zak 1968, Wannier 1979) when the flux through a unit cell is an irrational multiple of $2 \pi$ and the Hamiltonian of a system with an almost periodic potential (Simon 1982). It is now known that the density of states is in general discontinuous in the almost periods of the problem, e.g. for

$$
-\mathrm{d}^{2} / \mathrm{d} x^{2}+\cos x+\cos \alpha x=H
$$

the integrated density of states, $k(E, \alpha)$, [i.e. if $P_{(-\infty, E)}$ is the spectral projection for $H$ and $\chi_{(0, l)}$ multiplication by the indicator function of $(0, l)$ in $x$ space, then $k(E)=$ $\left.\lim _{l \rightarrow \infty} l^{-1} \operatorname{Tr}\left(P_{(-\infty, E) X(0, l)}\right)\right]$ is in general discontinuous at rational values of $\alpha$ and continuous at the irrational values of $\alpha$ (Avron and Simon 1982). This might suggest that the density of states is discontinuous in the strength of the magnetic field at rational values of flux. Our goal in this paper is to explain why gauge invariance implies that there is continuity of the density of states in a magnetic field. We give the argument in two space dimensions for notational convenience. It obviously extends to higher dimensions.

To be precise, fix a function, $V$, in two variables obeying $V(x+1, y)=V(x, y+1)=$ $V(x, y)$. Let

$$
H(B)=\left(-\mathrm{i} \partial_{x}+\frac{1}{2} B y\right)^{2}+\left(-\mathrm{i} \partial_{y}-\frac{1}{2} B x\right)^{2}+V(x, y) .
$$

Let

$$
k(E, B)=\lim _{l \rightarrow \infty} l^{-2} \operatorname{Tr}\left(P_{(-\infty, E)}(H(B)) \chi_{l}\right)
$$

where $\chi_{l}$ is the characteristic function of $|x| \leqslant \frac{1}{2} l,|y| \leqslant \frac{1}{2} l$; (the existence of the limit will be discussed below). It is somewhat easier to study

$$
\mathscr{L}(t, B)=\lim _{l \rightarrow \infty} l^{-2} \operatorname{Tr}\left(\mathrm{e}^{-t H(B)} \chi_{l}\right)
$$


and deduce properties of $k$ from $\mathscr{L}$. Let $P\left(t ; x, y ; x^{\prime}, y^{\prime}, B\right)$ be the integral kernel of $\mathrm{e}^{-t H(B)}$. For fixed $x, y$, it is easy to prove continuity in $B$, say, by exploiting the path integral formula for $P$ (Simon 1979). The key question is the uniformity of this continuity in $B$ near spatial infinity. Indeed, it is lack of this in the AP case that allows lack of continuity of $\mathscr{L}$ in $\alpha$.

The key observation is that while non-diagonal elements of $P$ are gauge dependent, diagonal elements are gauge independent and thus $P(t ; x, y ; x, y, B)$ is periodic in $x, y$. (In general, $P\left(t ; x+1, y ; x^{\prime}+1, y^{\prime}\right)$ differs from $P\left(t ; x, y ; x^{\prime}, y^{\prime}\right)$ by a phase factor equal to the flux through the parallelogram with vertices $(x, y),\left(x^{\prime}, y^{\prime}\right),(x+1, y)\left(x^{\prime}+1, y\right)$.) Thus, the limit in (2) exists and equals

$$
\int_{\substack{|x| \leqslant 1 / 2 \\|y| \leqslant 1 / 2}} P(t ; x, y ; x, y, B) \mathrm{d} x \mathrm{~d} y
$$

and this quantity is obviously continuous in $B$. To get back to $k$, one needs the following standard result from measure theory (Billingsky 1968).

Lemma. Let $\mathrm{d} \mu_{n}, \mathrm{~d} \mu_{\infty}$ be measures on $[0, \infty)$. Let $L_{n}(t)=\int \mathrm{e}^{-t x} \mathrm{~d} \mu_{n}(x)$ and let $k_{n}(E)=$ $\int_{-\infty}^{E} \mathrm{~d} \mu_{n}(x)$. Suppose $L_{n}(t) \rightarrow L(t)$ for each $t>0$. Then $(a) k_{\infty}(E)$ is continuous in $E$ at all but countably many $E$ and $(b)$ at any point $E$ where $k_{\infty}$ is continuous, $k_{n}(E) \rightarrow k(E)$.

Combining this with the arguments before we have:

Theorem 1. For any $B$, the limit (1) exists at all $E$ except for a countable set. If $B_{m} \rightarrow B$, then $k\left(E, B_{m}\right) \rightarrow k(E, B)$ for all but countably many $E$.

Alternatively, one could say the measures $\mathrm{d} k(\cdot, B)$ are continuous in $B$ in the weak topology on measures (Billingsky 1968).

In the exact same way, one proves the following theorem.

Theorem 2. Let $\boldsymbol{a}$ be a vector potential whose curl $\partial_{x} a_{y}-\partial_{y} a_{x}$ is periodic in $x$ and $y$. Let

$$
H(\lambda)=\left(-\mathrm{i} \partial_{x}-\lambda a_{x}\right)^{2}+\left(-\mathrm{i} \partial_{y}-\lambda a_{y}\right)^{2}
$$

and let $k(E, \lambda)$ be the corresponding integrated density of states. Let $\lambda_{m} \rightarrow \lambda$. Then for all but countably many $E, k\left(E, \lambda_{m}\right) \rightarrow k(E, \lambda)$.

We conclude with the resolution of an apparent paradox. In a strong-coupling approximation (Hofstader 1976), the potential $V(x, y)=\cos (x)+\cos (y)$ is equivalent to the finite-difference operator

$$
(h u)(n)=u(n+1)+u(n-1)+2 \cos (2 \pi \alpha n) u(n) .
$$

Yet the density of states for $n$ is discontinuous in $\alpha$ while the magnetic field density of states is continuous by the results here. The resolution of this we owe to $D$ Thouless, who remarked that the correct strong-coupling limit is a direct integral over $\theta$ of the operators

$$
(h(\theta) u)(n)=u(n+1)+u(n-1)+2 \cos (2 \pi \alpha n+\theta) u(n)
$$

and (Avron and Simon 1982) $\int_{0}^{2 \pi} k(\alpha, \theta) \mathrm{d} \theta$ is continuous in $\alpha$.

It is a pleasure to thank S Novikov for raising the questions answered here, and D Thouless for a valuable discussion. 


\section{References}

Avron J and Simon B 1982 Caltech preprints

Billingsky P 1968 Convergence of Probability Measures (New York: Wiley)

Hofstader D 1976 Phys. Rev. B 142239

Simon B 1979 Functional Integration and Quantum Physics (New York; Academic)

1982 Adv. Appl. Math. to be published

Wannier G et al 1979 Phys. Status Solidi b 93337

Zak J 1968 Phys. Rev. 168686 